OPEN ACCESS

Edited by: Aniruddh Sarkar, Georgia Institute of Technology, United States

Reviewed by: Tugba Önal-Süzek, Muğla University, Turkey Amr Ahmed El-Arabey, Al-Azhar University, Egypt

${ }^{*}$ Correspondence: Kai Ye

yekai1972@fimu.edu.cn

Specialty section:

This article was submitted to Human and Medical Genomics, a section of the journal Frontiers in Genetics

Received: 16 September 2021 Accepted: 28 December 2021 Published: 11 February 2022

Citation: Chen J, Liu W, Du J, Wang P, Wang J and Ye $K$ (2022) Comprehensive Genomic and Epigenomic Analyses on Transcriptomic Regulation in Stomach Adenocarcinoma.

Front. Genet. 12:778095 doi: 10.3389/fgene.2021.778095

\section{Comprehensive Genomic and Epigenomic Analyses on Transcriptomic Regulation in Stomach Adenocarcinoma}

\author{
Junxing Chen, Weinan Liu, Jiabin Du, Pengcheng Wang, Jintian Wang and Kai Ye* \\ Department of Oncology Surgery, Second Affiliated Hospital of Fujian Medical University, Quanzhou, China
}

Background: DNA methylation (MET)-mediated transcriptomic disturbance and copy number variations (CNVs) exert a significant influence in stimulating the heterogeneous progression of stomach adenocarcinoma (STAD). Nevertheless, the relation of DNA MET with CNVs, together with its impact on tumor occurrence, is still unclear.

Methods: The messenger RNA (mRNA) expression (EXP) profiles, DNA MET, and DNA copy numbers, together with STAD mutation data, were collected from the TCGA official data portal. We employed circular binary segmentation algorithm in "DNAcopy." library of $R$ package for mapping DNA CNV data at genetic level for all samples based on the segmented CNV data. Stable clusters of samples were recognized using negative matrix factorization cluster analysis based on 50 iterations and the "brunet" method using the MET-correlated (METcor) and CNV-correlated (CNVcor) genes. The R package "iCluster" method was utilized to comprehensively analyze the EXP, MET, and DNA CNV profiles.

Results: A total of 313 STAD samples were isolated for checking DNA copy numbers and MET and for measuring EXP. In accordance with our results, we discovered obvious coregulation of CNVcor genes and METcor counterparts. Apart from that, these genes were subject to multi-omics integration. Meanwhile, three subtypes of STAD were detected and confirmed based on independent data. Among them, the subtype with increased aggressiveness was related to decreased mutation frequencies of ARID1A, PIK3CA, ZFHX3, SPECC1, OBSCN, KMT2D, FSIP2, ZBTB20, TTN, and RANBP2, together with the abnormal levels of JPH3, KCNB1, and PLCXD3.

Conclusion: According to the results, these aforementioned genes exerted crucial roles in the development of invasive STAD. Our findings on transcriptomic regulation genomically and epigenetically facilitate the understanding of the STAD pathology from different aspects, which help to develop efficient anti-STAD therapy.

\footnotetext{
Abbreviations: CNV, copy number variation; CNV cor, CNVs-correlated; GC, gastric cancer; ER, endoplasmic reticulum; JMC junctional membrane complex; MET, methylation; METcor, MET-correlated; mRNA, messenger RNA; NMF, negative matrix factorization; PM, plasma membrane; TNM, tumor node metastasis; STAD, stomach adenocarcinoma.
} 
Keywords: stomach adenocarcinoma, integrative analysis of genomic and epigenomic regulation, DNA copy number-correlated genes, methylation-correlated genes, mutation-genetics

\section{BACKGROUND}

Gastric cancer (GC), the cancer with high malignancy rate, accounts for about $7.7 \%$ of cancer-related deaths in the world in 2020. Related treatments have been developed for GC. However, it is still the fatal malignant tumor that ranks the fourth place in terms of its mortality globally, which is ascribed to its advanced diagnosis and high morbidity (Sung et al., 2021). Typically, the overall survival (OS) rate of GC at 5 years is less than $25 \%$, especially for the recurrent and advanced types (Bray et al., 2018). Stomach adenocarcinoma (STAD) takes up about 90\% of global GC cases (Bu and Ji, 2013; Karimi et al., 2014). Most STAD patients among western countries are diagnosed at the metastatic or advanced stage (Van Cutsem et al., 2011). Although great advances have been attained in chemotherapy, radiotherapy, and surgery, prognosis of STAD still remains dismal. Some GC patients present diverse prognostic outcomes and therapeutic responses, even though they are at the same TNM (Tumor Node Metastasis) stage (Shiratsu et al., 2014). Early STAD diagnosis markedly enhances patient outcomes. As a result, the biomarkers for diagnosis and prognosis are in urgent need to improve the STAD diagnosis and to predict the outcomes for patients.

Markers for diagnosis and prognosis can be used to closely monitor and treat the high-risk patients, so as to extend their OS time. The common STAD biomarkers applied in clinic are mainly the clinicopathological parameters, such as tumor stage, age, chemotherapy response, and infection with Helicobacter pylori (Ye et al., 2004). Developing new biomarkers can introduce and design new therapeutic strategies for improving the survival for patients. Therefore, comprehensive examination of the disease molecular characteristics is of crucial necessity.
In recent years, the huge multi-omics greatly contributes to comprehensively displaying disease dysregulation at genomic and epigenetic levels (Woo et al., 2017). Genomic alternations induced by cancer filing, such as DNA mutations and copy number variations (CNVs), are common during tumor genesis, which promote cancer progression (Shi et al., 2016). In addition, cancer genomic regulation via DNA methylation (MET) at an epigenetic level exerts a crucial part in the behaviors of various cancers, including STAD (Peng et al., 2020; Song et al., 2020). Genomic profiling research suggests that genomic and epigenomic dysregulation is highly heterogeneous. DNA CNVs exert a vital part in STAD for regulating STAD development; in addition, the resultant transcriptional dysregulation is potentially a driving event during the progression of STAD (Feng et al., 2019; Cai et al., 2020). Besides, research on DNA MET profiling suggests that epigenetic regulation is highly significant in the development of cancer from the points of view of biology and clinic (Choi et al., 2017; Ebrahimi et al., 2020). At the same time, some critical tumor-associated genes, such as RASSF1A, SAMD14, and SOCS3, can modulate DNA MET, thereby regulating their functions (Balgkouranidou et al., 2015; Han et al., 2020; Xu et al., 2020).

Nonetheless, the association between DNA MET and CNVs is still unknown, even though DNA MET alternations and CNVs affect the whole genome of cancer. Besides, the impact of this association during cancer development remains unclear. In the current work (flowchart is shown in Figure 1), we extracted STAD samples for analyzing DNA copy numbers and MET, together with the messenger RNA (mRNA, EXP) expression. In addition, genes related to DNA copy numbers (CNV cor) and MET (METcor) were identified according to related gene levels in the aforementioned samples, separately, so as to recognize genes with genomically and/ or epigenetically modulated expression. Of them, CNVcor genes

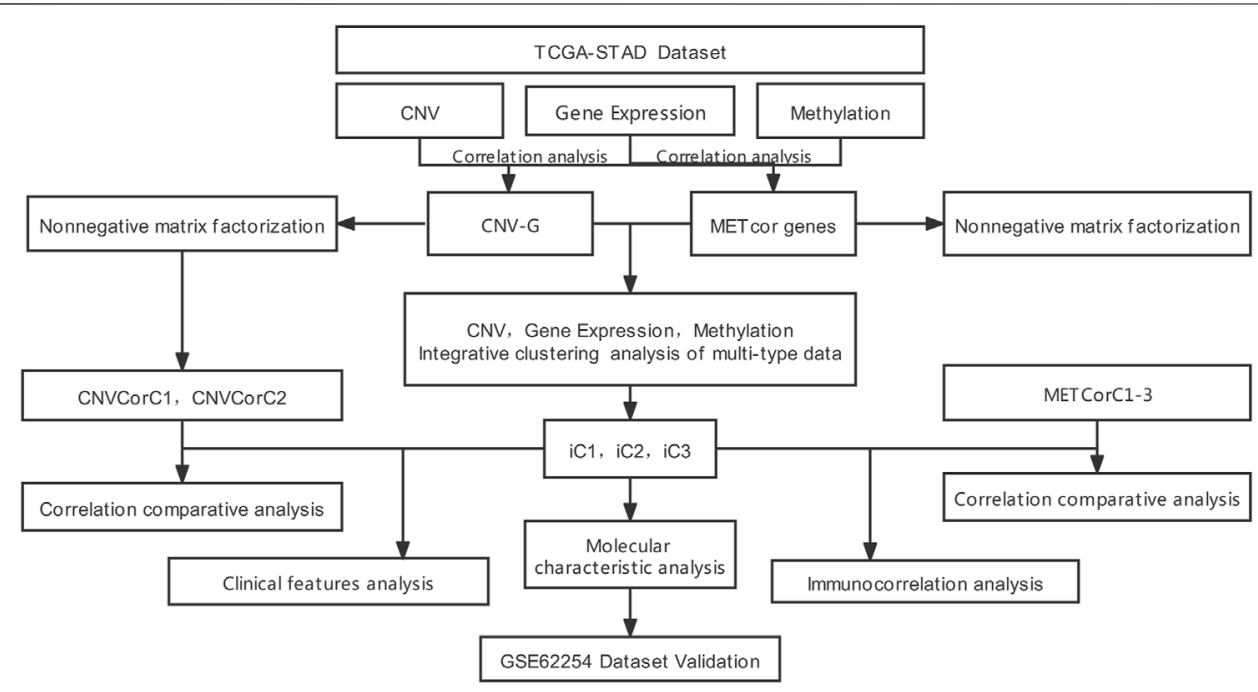

FIGURE 1 | The work flow chart of this study. 


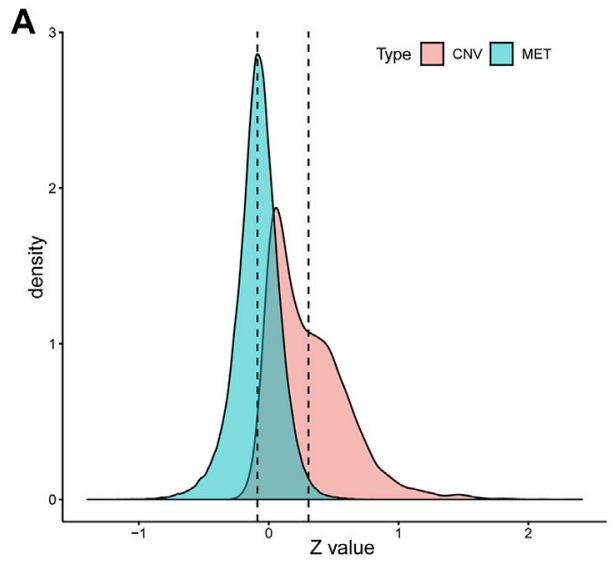

B

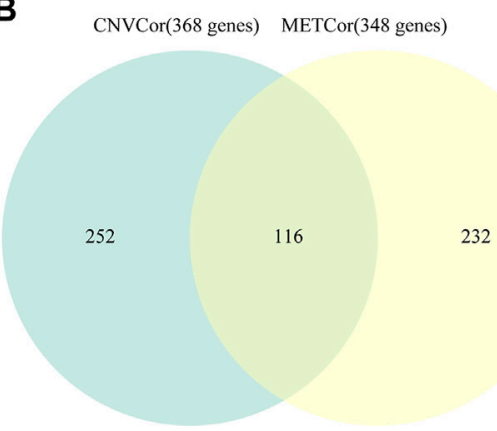

C

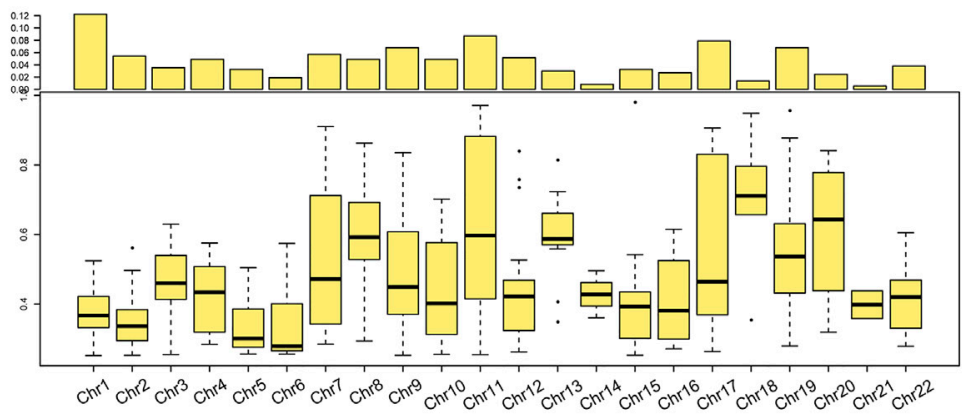

D
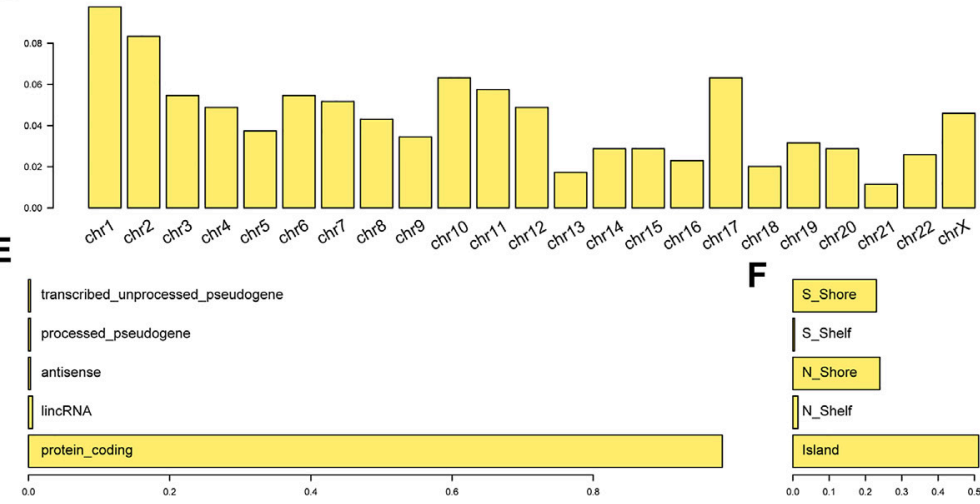

FIGURE 2 | Recognition of DNA methylation-correlated (METcor) and DNA copy number-correlated (CNVcor) genes of STAD. (A) Correlation coefficient distribution of mRNA expression with DNA MET or DNA copy numbers among different specimens is presented, respectively. (B) The Venn diagram displays the METcor and CNVcor gene numbers. Overlapped gene number between METcor and CNVcor genes is indicated. (C) Proportions of CNVcor genes in the total number of genes in every chromosome arm. (D) METcor gene proportions in the overall number of genes in every chromosome arm. (E,F) Genomic locations of DNA MET probes were classified according to the relationships with genes (left) and CpG islands (right) in positions, separately.

suggested transcriptional dysregulation according to DNA copy numbers, while METcor ones represented transcriptional dysregulation according to DNA MET. CNVcor gene levels were evidently related to METcor gene levels, which indicated cancer transcriptomic co-regulation via genomic DNA CNVs and epigenetic DNA MET abnormalities. Besides, we conducted multiomics integration of METcor and CNVcor genes, so as to examine the typical molecular subtypes to predict the prognosis of STAD. Furthermore, we also supplied the differential and correlation analyses on tumor microenvironment and immune infiltration among different subtypes and different samples, which have improved the guiding significance for the individualized immunotherapy for GC patients. Novel specific targets and biomarkers to distinguish different subtypes of cancer were identified through intensive systematic analysis.

\section{METHODS}

\section{mRNA Expression Patterns, DNA MET, and DNA Copy Numbers}

A STAD dataset was downloaded from TCGA for analyzing related results. The mRNA expression profiles, DNA MET, and DNA copy numbers, together with STAD mutation data, were collected from the TCGA official data portal. Each sample mark was matched in every platform; afterwards, altogether 313 datasets were utilized (Supplementary Table S1), which had corresponding datasets for mRNA expression profiles, DNA MET, and DNA copy numbers. In addition, gene expression patterns were standardized through $\log 2$ transformation and quantile normalization, followed by HUGO official symbol aggregation. The expression profiles were subsequently standardized through eliminating the average values from each probe within non-cancer tissues, which stood for the fold change in tumor samples relative to healthy samples. Thereafter, we utilized the $\mathrm{R}$ package circular binary segmentation algorithm in "DNAcopy." library for mapping DNA CNV data at the genetic level for all samples based on the segmented CNV data (Zhang et al., 2017). With regard to the DNA MET profiles, the $\beta$-values of the probe were filtered for eliminating probes positioned on the sex chromosomes. Later, probes located in the regions associated with the $\mathrm{CpG}$ islands were mapped to associated genes, such as Shore regions, CpG islands, Shelf, differentially methylated regions, first-exon regions, $5^{\prime} \mathrm{UTR}$, and the gene promoter areas that contained 2,500 upstream basic groups from TSS. We eliminated probes that had over $30 \%$ missing values among the samples from every processed pattern. 


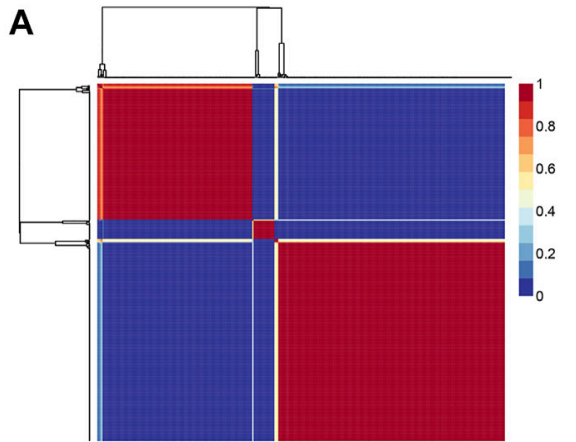

B

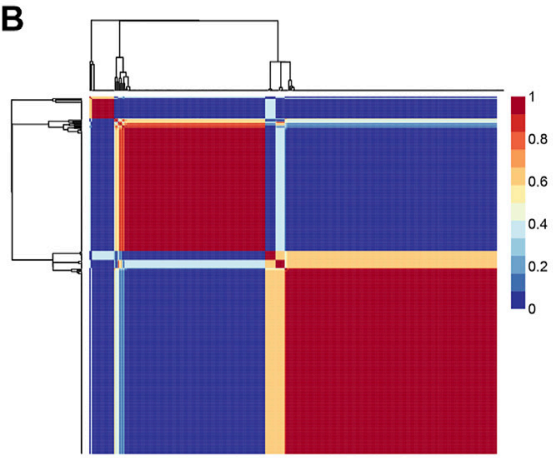

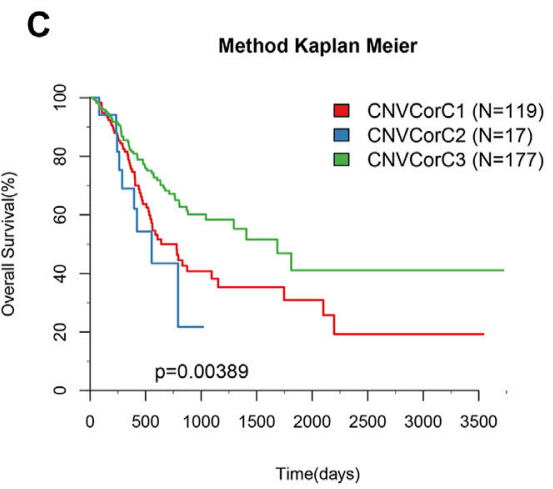

D

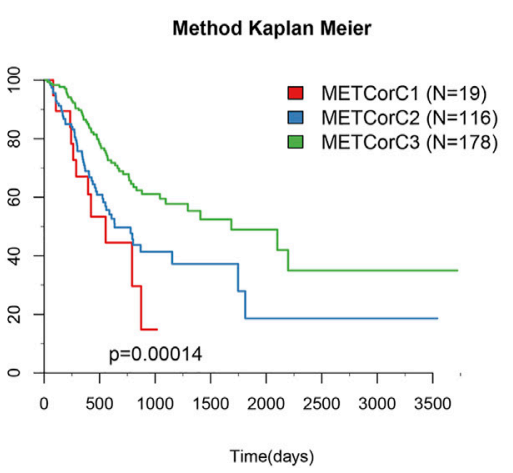

E

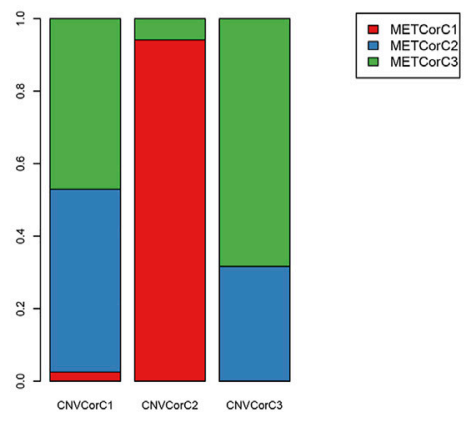

$\mathbf{F}$

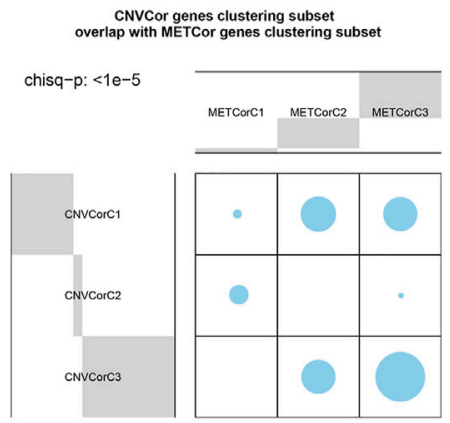

FIGURE 3 | Identification of STAD molecular subtypes by METcor and CNVcor genes. (A,B) Plots displaying cluster results of NMF of CNVcor gene from CNV data (A) and METcor genes from MET data (B), separately. (C,D) OS Kaplan-Meier curves for subtypes stratified based on NMF cluster analysis on CNVcor (C) and METcor (D) genes, separately. (E,F) Subtypes identified according to CNVcor show marked overlaps with those identified by METcor.

The "liftOver," library of R package was utilized for every dataset, so as to restore the genomic coordinates of probe against human reference genome hg38. Afterwards, we matched the probes to related ones from EXP profiles. Cancer-specific alterations were computed through eliminating the mean probe intensity within non-cancer tissues. Then, probes on the sex chromosomes and those that had over 50\% missing values were eliminated, and related information was input with the sknearest neighbor algorithm. Thereafter, we calculated the pairwise Pearson's correlation coefficients of all genes across the matched EXP and CNV profiles, as well as EXP and MET, respectively. If over one probe was mapped to one gene, the probe that had the average or the smallest correlation coefficient was utilized to be the typical pair-matched probes of MET and CNV profiles, separately.

\section{Cluster Analysis on the Genomic Patterns at Different Levels}

Stable clusters of samples were recognized by cluster analysis of negative matrix factorization (NMF) based on 50 iterations and the "brunet" method through CNVcor and METcor genes, separately (Zeng et al., 2019). Notably, we set k as 2-10 and calculated the best $\mathrm{k}$ according to the observed consensus map, together with the cluster cophenetic correlation. At the same time, to examine consensus membership matrix, the mean silhouette width was computed by "NMF." of R package. Regarding all membranes, we set the lowest $k$ as 10 . Then, we utilized R package "iCluster" method for comprehensively analyzing the EXP, MET, and DNA CNV profiles; besides, 20 iterations and the default parameters were utilized (Shen et al., 2012).

\section{Evaluation and Identification of Immune Cell Infiltration}

In this study, we identified and evaluated the abundance of immune infiltrates by the TIMER algorithm, which is a resource for systematic and extensive analysis of immune infiltrates (totally six cell types, namely: B cells, $\mathrm{CD}^{+} \mathrm{T}$ cells, $\mathrm{CD}^{+} \mathrm{T}$ cells, neutrophils, macrophages, and dendritic cells) across diverse cancer types.

\section{RESULTS}

\section{Transcriptomic Alternations in DNA Copy Number or DNA MET}

DNA CNVs and MET at genetic and epigenomic levels, as well as gene EXP patterns, were collected from 313 STAD specimens. Then, the raw materials were preprocessed according to the method described previously in "Methods". Later, the correlation coefficients of DNA CNV or MET profiles with corresponding mRNA EXP data were computed, so as to evaluate the influence of epigenomic and/or genomic 


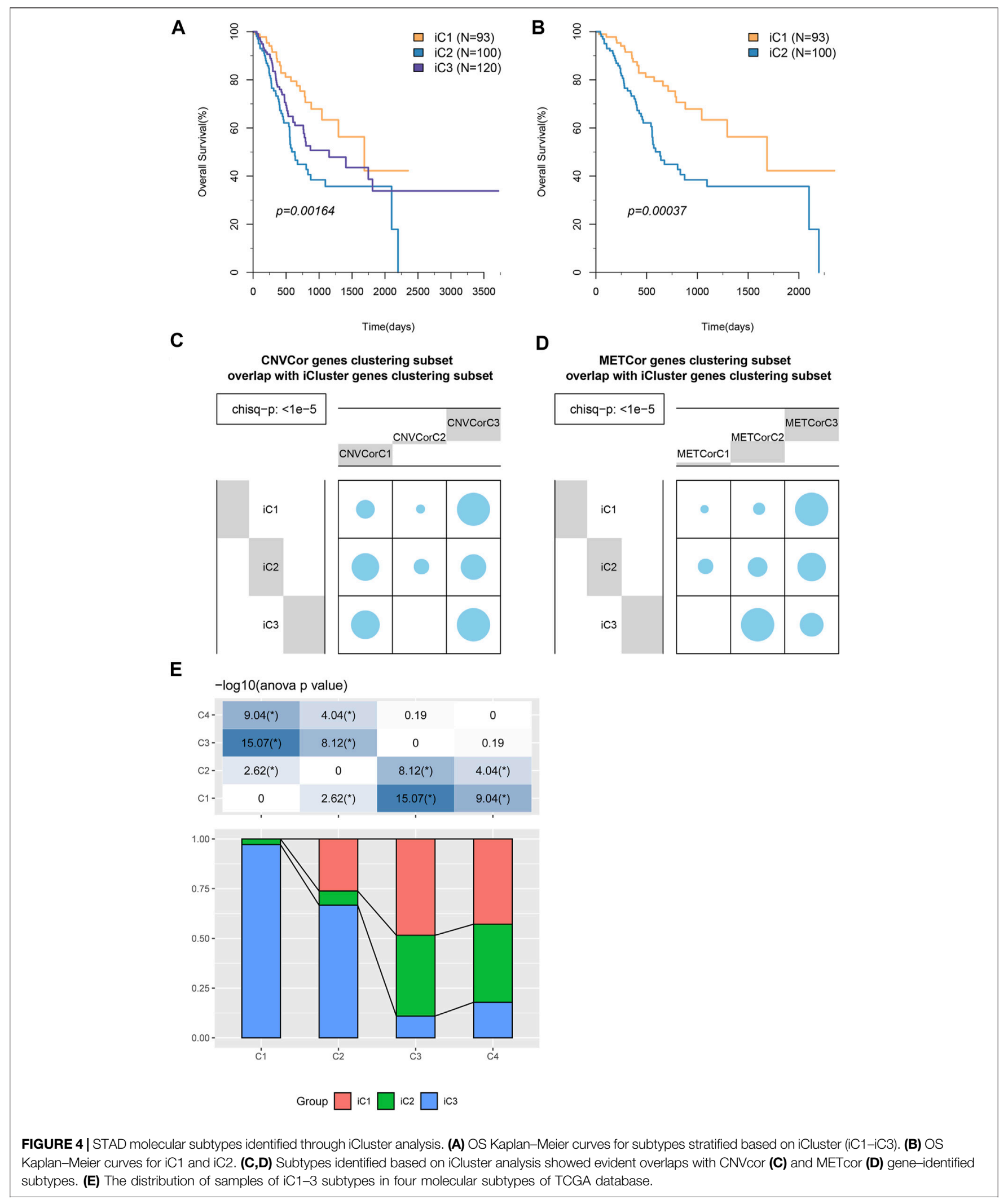



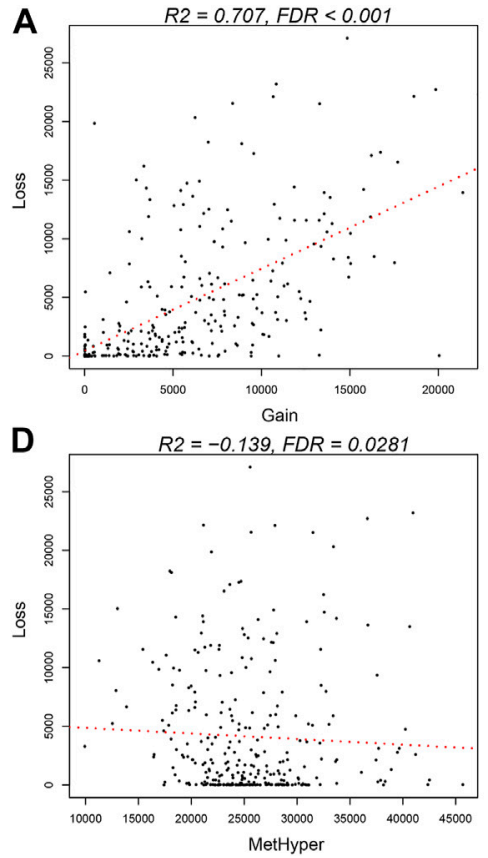
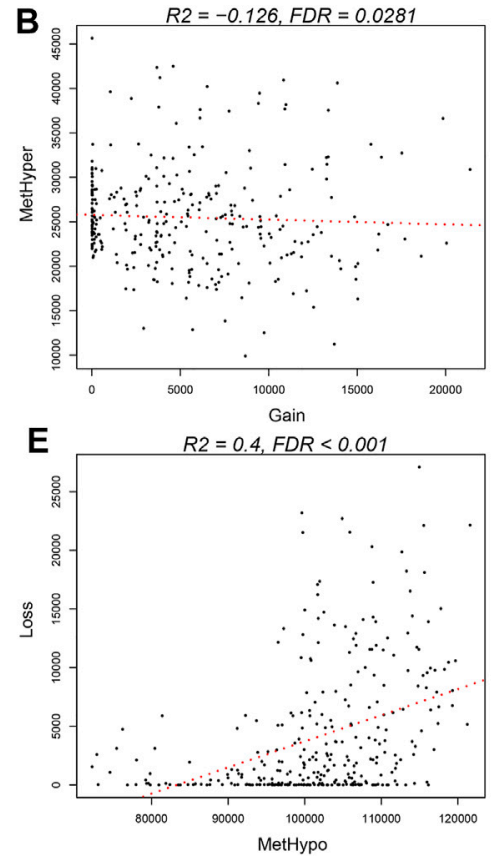

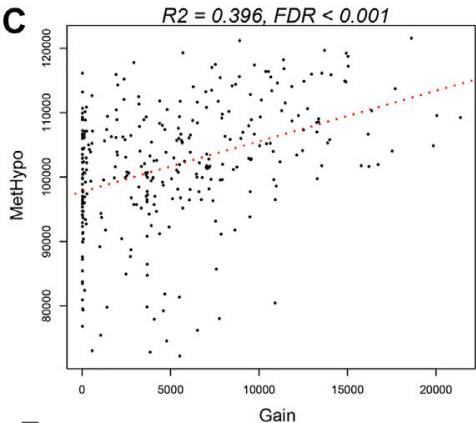

$\mathbf{F}$

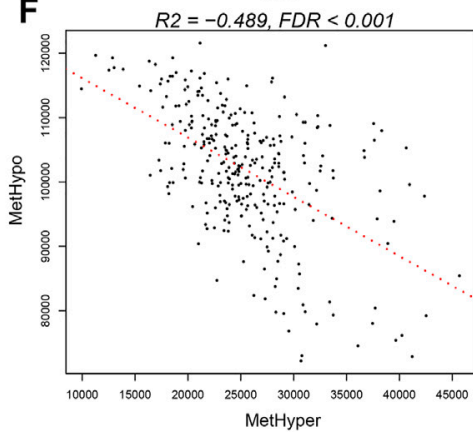

FIGURE 5 | Coordinated DNA MET abnormalities and DNA CNVs in STAD. (A,F) DNA CNV or DNA MET abnormalities were identified at the threshold fold change of $>0.2$ relative to the means in normal tissues, respectively. Directional DNA CNV-gain (CNVgain) and CNV-loss (CNVloss), together with DNA hypomethylation (METhypo) and hypermethylation (METhyper), in all samples are presented, respectively. (B-E) Plots display pairwise occurrence rates of CNVloss, CNVgain, METhypo, and METhyper genes in each sample.

abnormalities. Thereafter, we standardized the correlation coefficient $r$ according to Fisher's Z-transformation for variance stabilization.

Consistent with a prior study, correlation coefficient distribution of DNA CNV with related EXP data markedly skewed to the right (skewness $=1.2352, p<1 \mathrm{e}^{-5}$ ). In comparison, the correlation coefficients of DNA MET with related EXP data skewed to the left (skewness $=-0.37363, p<$ $1 \mathrm{e}^{-5}$ ) (Figure 2A), which indicated that DNA CNVs and MET abnormalities positively and negatively modulated transcription, respectively.

A great number of genes participated in the two aforementioned gene sets (CNVcor and METcor genes, Supplementary Tables S2, S3); as a result, we selected genes that were markedly correlated with OS for later analyses (log rank $p<0.05)$. Then, we screened gene signatures showing positive correlation to examine DNA copy number (CNVcor, $n=368$ ) and those showing negative correlation for DNA MET (METcor, $n=348)$. CNVcor genes indicated that transcriptional dysregulation was dependent on DNA CNVs, but METcor genes suggested that it was dependent on MET. CNVcor genes were not overlapped with METcor ones; of them, 116 overlapped genes were identified, which suggested that CNVcor and METcor genes specifically regulated transcriptional dysregulation (Figure 2B).

CNVcor genes preferred DNA CNVs within some genomic regions, especially on chromosomes 10, 17, 18, and 21 (Figure 2C and Supplementary Table S4). Consistent with prior research,
CNVcor genes were abundant on chromosomes 10 and 17, which suggested that gene expression was sensitive to DNA level in certain regions (Arakawa et al., 2017). In addition, METcor genes were found within desired chromosomal regions, like chromosome 19 (Figure 2D and Supplementary Table S5), most of which were genes that encoded proteins (Figure 2E) and were distributed in the CpG islands (Figure 2F). This study suggested that $\mathrm{CNV}$ cor and METcor genes were remarkably effective on transcriptional dysregulation of STAD, which required to be further examined in future studies.

\section{Different CNVcor and METcor Gene-Dependent Molecular Subtypes}

Subsequently, the effects of CNVcor and METcor gene expression on predicting prognostic subgroups were explored. NMF cluster analysis was performed for all gene set data, and we set $\mathrm{k}$ as 2-10; later, $k$ value was calculated for each profile $(k=3$ for $\mathrm{CNV}$ and MET, respectively) (Figures 3A,B). Surprisingly, CNVcor gene-identified subtypes were remarkably overlapped with the METcor gene-identified ones ( $p<1 \mathrm{e}^{-5}$ upon $\chi^{2}$ test), and such results were consistent with $\mathrm{CNV}$ cor and METcor gene regulation in STAD (Figures 3E,F). In addition, Kaplan-Meier (KM) curve results suggested that CNVcor or METcor gene-identified subtypes predicted the OS of patients (Figures 3C,D), separately $(p<0.05)$.

Molecular subtypes associated with CNVcor and METcor gene expression were identified at various aspects. iCluster, the 
TABLE 1 | Clinical information of STAD patients across the three subtypes were compared

\begin{tabular}{|c|c|c|c|c|}
\hline Event & Total & iC1 & iC2 & iC3 \\
\hline Alive & 188 & 69 & 47 & 68 \\
\hline Dead & 125 & 22 & 49 & 49 \\
\hline \multicolumn{5}{|l|}{$\mathrm{T}$} \\
\hline T1 & 14 & 7 & 5 & 2 \\
\hline T2 & 63 & 17 & 26 & 20 \\
\hline T3 & 146 & 41 & 47 & 58 \\
\hline $\mathrm{T} 4$ & 81 & 26 & 18 & 37 \\
\hline \multicolumn{5}{|l|}{$N$} \\
\hline NO & 90 & 35 & 24 & 31 \\
\hline N1 & 80 & 20 & 25 & 35 \\
\hline N2 & 65 & 20 & 22 & 23 \\
\hline N3 & 62 & 14 & 23 & 25 \\
\hline $\mathrm{NX}$ & 7 & 2 & 2 & 3 \\
\hline \multicolumn{5}{|l|}{ M } \\
\hline MO & 275 & 87 & 86 & 102 \\
\hline M1 & 18 & 2 & 5 & 11 \\
\hline$M X$ & 11 & 2 & 5 & 4 \\
\hline \multicolumn{5}{|l|}{ Stage } \\
\hline 1 & 39 & 14 & 14 & 11 \\
\hline$\|$ & 99 & 34 & 27 & 38 \\
\hline III & 129 & 36 & 40 & 53 \\
\hline IV & 28 & 3 & 13 & 12 \\
\hline Un & 9 & 4 & 2 & 3 \\
\hline \multicolumn{5}{|l|}{ Grade } \\
\hline G1 & 8 & 2 & 3 & 3 \\
\hline G2 & 107 & 37 & 49 & 21 \\
\hline G3 & 180 & 51 & 40 & 89 \\
\hline$G X$ & 9 & 1 & 4 & 4 \\
\hline \multicolumn{5}{|l|}{ Age (years) } \\
\hline $0-50$ & 27 & 9 & 7 & 11 \\
\hline $50-60$ & 72 & 15 & 20 & 37 \\
\hline $60-70$ & 91 & 23 & 33 & 35 \\
\hline $70-80$ & 99 & 38 & 32 & 29 \\
\hline $80-100$ & 15 & 6 & 4 & 5 \\
\hline \multicolumn{5}{|l|}{ Gender } \\
\hline Female & 101 & 29 & 27 & 45 \\
\hline Male & 203 & 62 & 69 & 72 \\
\hline
\end{tabular}

integrated cluster method, was employed to integrate genomic data on mRNA EXP, DNA CNVs, and MET. Afterwards, cluster analysis was carried out with the cluster number $\mathrm{k}$ of $2-4$. Altogether, 20 cluster iterations at $\mathrm{K}=4$ (category 5), $\mathrm{K}=3$ (category 4 ), and $\mathrm{K}=2$ (category 3 ) were carried out separately to assess the best iCluster cluster results. According to our findings, stable cluster results were obtained at $\mathrm{K}=2$ relative to those at $\mathrm{K}=$ 3 or 4 (Supplementary Figures S1, S2). As a result, all samples were clustered into three subclasses of $\mathrm{iC} 1-\mathrm{iC} 3(n=93,100$, and 120, respectively). Supplementary Figures S3A,B display the cluster results of those three subclasses, and Supplementary Table S6 presents those of all samples.

$\mathrm{KM}$ analysis results suggested that $\mathrm{iC} 1$ attained the optimal OS across those three subtypes ( $p<0.05$, Figure 4A). The OS of patients in $\mathrm{iC} 1$ subgroup was compared with that of the other two subgroups (Figure 4B and Supplementary Figure S4), and the results suggested that the difference in prognosis between $\mathrm{iC} 1$ and iC2 subgroups was statistically significant $(p<0.001)$. Notably, those icluster-identified subtypes remarkably overlapped with CNVcor and METcor gene-identified counterparts $\left(p<1 \mathrm{e}^{-5}\right.$, $\chi^{2}$ test, Figures 4C,D). In addition, we have explored the distribution of samples of iC1-3 subtypes in four molecular subtypes of TCGA database, and the results (Figure 4E) suggested that samples of iC3 subtype were mainly distributed into $\mathrm{C} 1$ and $\mathrm{C} 2$ subtypes, while samples of $\mathrm{iC} 2$ subtype were mainly distributed in $\mathrm{C} 3$ and $\mathrm{C} 4$ subtypes. According to the aforementioned findings, comprehensive analysis on CNVcor and METcor genes facilitated to detect various molecular subtypes, and all of them showed heterogeneous combinations of genomic and epigenomic features associated with prognosis and transcriptional dysregulation.

\section{Comparisons of DNA CNV and MET Abnormalities}

The frequencies between genome-wide DNA MET abnormalities and DNA CNVs were compared after batch effect correction. In addition, DNA copy-number gain (CNVgain, $\beta>0.3$ ) and loss (CNVloss, $\beta<-0.3$ ), together with DNA hypermethylation (METhyper, $\beta>0.8$ ) and hypomethylation (METhypo, $\beta<$ 0.2 ), were computed according to the determined threshold (fold change of 0.3 ), which were subsequently compared with the level of every probe. Our results indicated that (Supplementary Table S7) CNVgain frequency showed marked correlation with CNVloss frequency $\left(p<1 \mathrm{e}^{-5}\right.$, Figure 5A). Besides, METhyper frequency displayed evident correlation with METhypo frequency ( $p<1 \mathrm{e}^{-5}$, Figure 5F). Directional METhyper and CNVgain were tightly correlated with CNVloss, which indicated that all correlations were directional abnormality free (Figures 5B-E, $p<0.05$ ). In conclusion, our results suggested that STAD patients with increased frequency of DNA CNVs had elevated frequency of DNA MET abnormality. Correlations between the frequency of abnormal METcor and CNVcor genes indicated the close correlation of DNA MET with DNA CNVs.

\section{Identification of STAD Subtype Key Features}

First, clinical features (including TNM, stage, gender, and primary site) were compared across those three subtypes. Supplementary Figure S5 and Table 1 indicated that the differences in clinical characteristics were not statistically significant among three STAD subtypes. Nonetheless, as for stage distribution, III and IV samples mainly belonged to iC2 subtype that had the poorest prognosis. In addition, considering the significance of TCGA to study the infiltration and the complex interaction of immune cells within tumor microenvironment (TME) (El-Arabey et al., 2020), tumor immune microenvironment (TIME) status of samples across these three subtypes were also calculated and determined according to the tumor immune estimation resource (TIMER) method (Li et al., 2016) (Supplementary Table S8) (Li et al., 2016). As observed, differences in the $5 / 6$ immune cell scores $\left[\mathrm{CD}^{+} \mathrm{T}, \mathrm{CD}^{+} \mathrm{T}\right.$, macrophages, dendritic cells (DCs), 

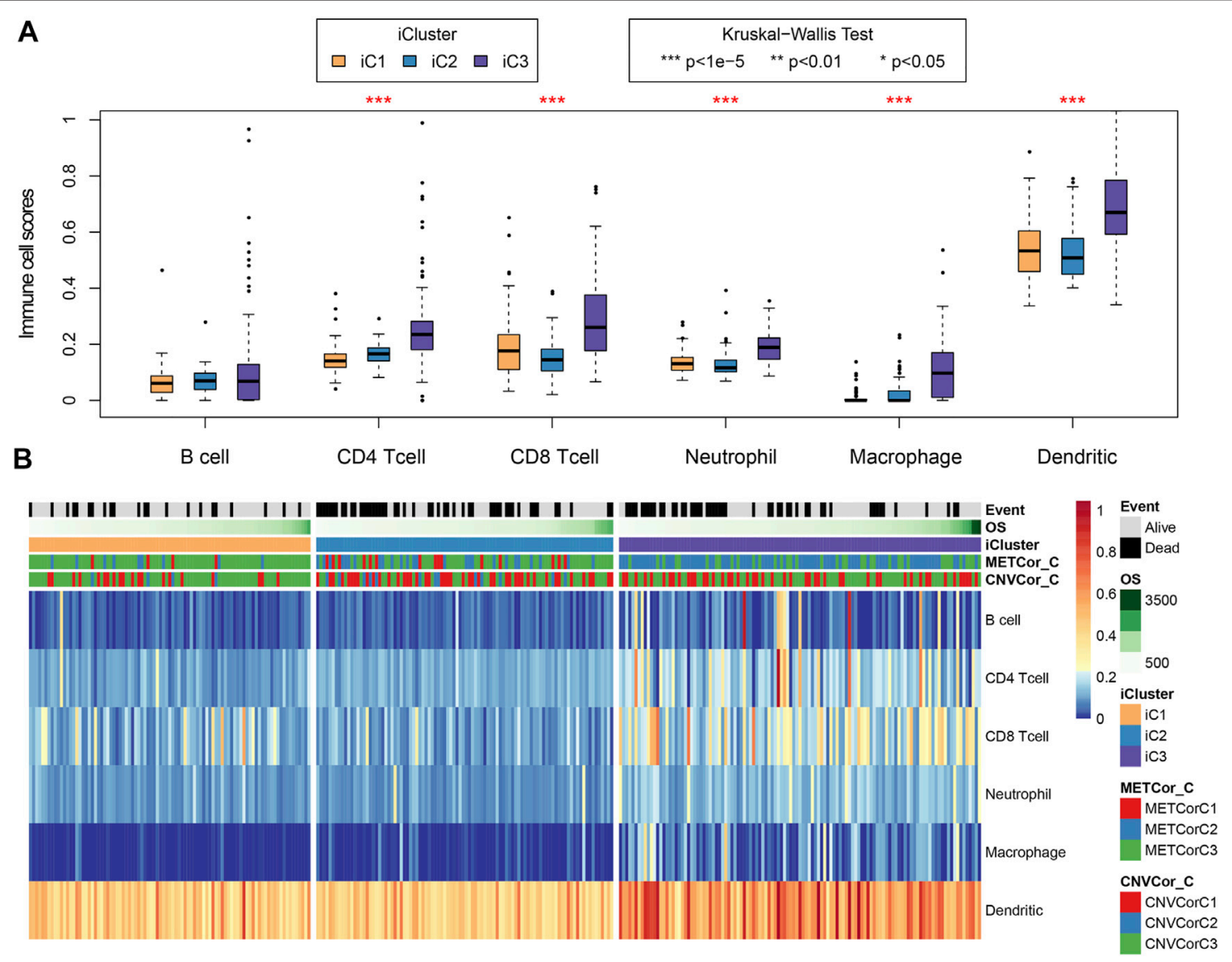

FIGURE 6 | Key STAD immune features. Sample immune scores among the three subtypes were computed and analyzed by the tumor immune estimation resource (TIMER) method. Scores of six immune cells in all samples were determined (B) and analyzed compared with those in samples of other subtypes (A).

neutrophils] of samples across these three subtypes were statistically significant (Figures 6A,B, $p<1 \mathrm{e}^{-5}$ ). The aforementioned results indicated that immunocyte infiltration degree or the immune microenvironment of STAD was correlated with DNA CNV or MET level.

Besides, differences in gene EXP, DNA CNVs, and MET between samples of $\mathrm{iC} 2$ and $\mathrm{iC} 1$ subtypes were compared. Later, DNA MET and CNV levels were classified as three types, including Normal, Gain, and Loss (for CNV), as well as Normal, HyperMethy and HypoMethy (for MET). DNA CNVcor or METcor genes with marked difference between $\mathrm{iC} 2$ and $\mathrm{iC} 1$ subtypes were obtained through Fisher's exact test. These findings can be observed from Supplementary Tables $\mathbf{S 9}$ and $\mathbf{S 1 0}$. As for EXP patterns, we acquired DEGs of $\mathrm{iC} 1$ versus $\mathrm{iC} 2$ subtypes through DESeq2 (Love et al., 2014) $(p<0.05)$, and the results are presented in Supplementary Table S11. In addition, to illustrate the crucial prognostic features across various subtypes, altogether five genes with distinct difference between $\mathrm{iC} 1$ and $\mathrm{iC} 2$ samples at all the three (MET, CNV, and EXP) levels were screened to carry out univariate survival analysis. According to our findings, two genes (PLCXD3 and KCNB1) were related to overall survival (OS) (log-rank $p<$ 0.05), suggesting that the two aforementioned genes in iC2 subtype (with poorer prognosis) had greater $\mathrm{CNV}$ and hypermethylation levels than iC1 subtype (with positive prognosis), and their levels within $\mathrm{iC} 1$ subtype were downregulated compared with those in the iC2 subtype. Afterwards, PLCXD3 and KCNB1 expression was divided as low, moderate, and high (L1-L3). Our findings suggested that L1-L3 groups with regard to PLCXD3 and KCNB1 expression levels showed positive correlation with OS (Figures 7A,B). The aforementioned findings indicated that the distinct PLCXD3 and KCNB1 expression was related to DNA MET or CNV level as well as the patient prognostic outcome. Moreover, the GEO GSE62254 (Cristescu et al., 2015) STAD dataset $(n=266)$ was utilized for analyzing the relation of those five genes with patient prognosis. The expression levels of JPH3 and KCNB1 were available, suggesting that only JPH3 in GSE62254 STAD dataset (Oh et al., 2018) showed marked correlation with prognosis. Supplementary Figure S6 displays the results. The relationship between JPH3 expression and sample OS in multiple GEO cohorts was also measured (http://kmplot.com/analysis/, Supplementary Figure S7). In addition, we determined the relationships of JPH3, PLCXD3, and KCNB1 levels with infiltrating degrees of immune cells within TME. As shown in Supplementary Figure S8, these three genes showed significant positive correlation with enrichment levels of most immune cells $\left(\mathrm{CD} 4^{+} \mathrm{T}, \mathrm{CD}^{+} \mathrm{T}\right.$, macrophages, DCs, neutrophils). 

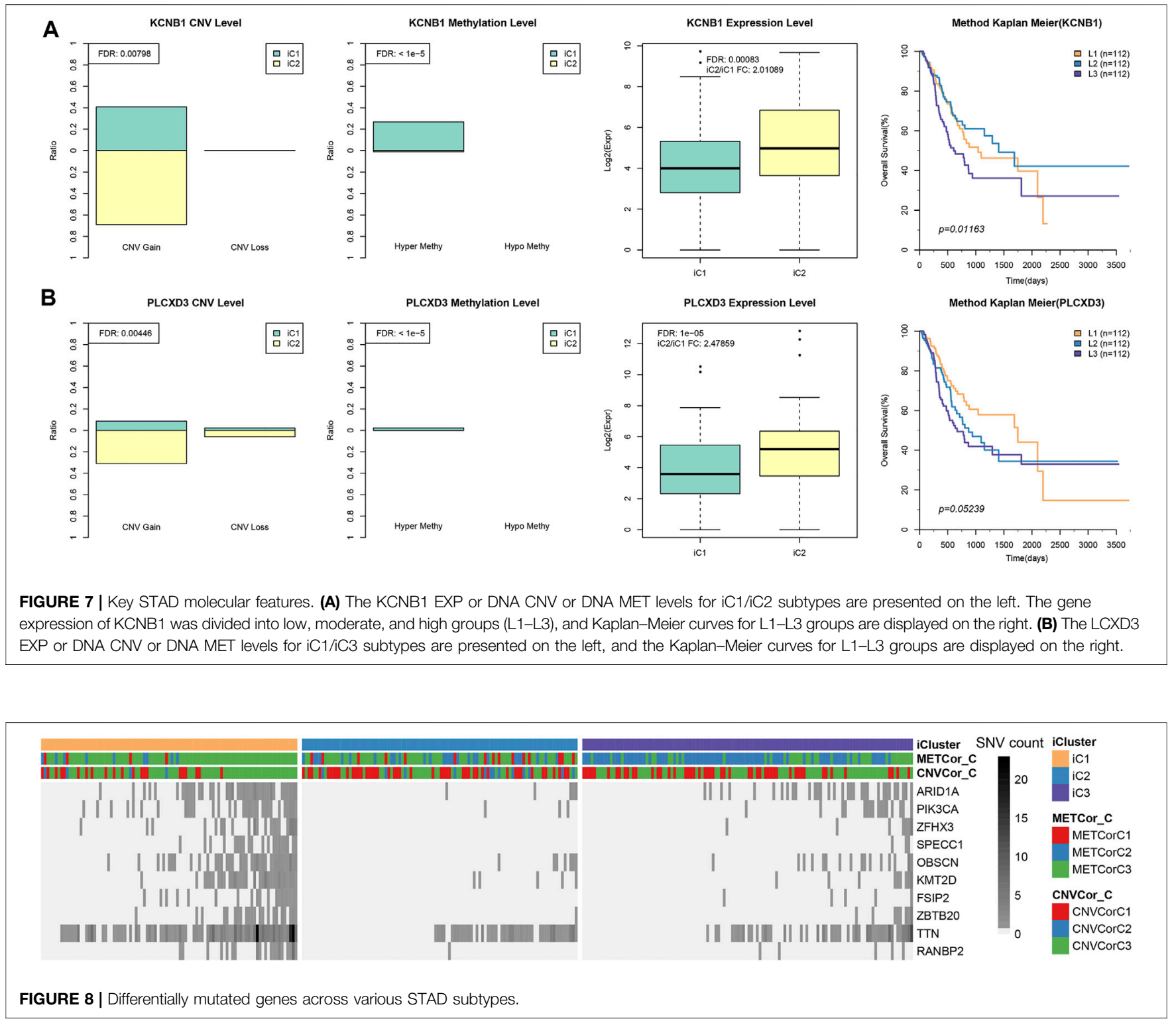

Eventually, the STAD mutation profiles were determined for exploring the associations with the sub-classification. Specifically, the synonymous mutations were eliminated to obtain the missense and nonsense mutations. Overall, the mutation frequency of genes in different subtypes showed statistically significant differences. In addition, some distinctly different mutant genes (ARID1A, PIK3CA, ZFHX3, SPECC1, OBSCN, KMT2D, FSIP2, ZBTB20, TTN, RANBP2) between $\mathrm{iC1}$ and $\mathrm{iC2}$ subtype were selected upon Fisher's test, as presented in Figure 8 and Supplementary Table S12 (FDR < 0, one upon Fisher's test). The different mutational spectra were analyzed, which suggested that the 10 genes in iCl subtype had remarkably increased mutation frequencies compared with those in $\mathrm{iC} 2$ and $\mathrm{iC} 3$ subtypes $(p<0.01)$. Interestingly, mutation frequencies of these 10 genes in iC3 subtype with common prognosis also increased relative to iC2 subtype $(p<0.05)$. Collectively, the aforementioned findings indicated that the STAD molecular subtypes related to DNA copy numbers and DNA MET showed correlation with mutations in the 10 genes, and they might modulate the progression of STAD subtypes.

\section{DISCUSSION}

Prior studies indicate that comprehensive analysis on tumor genomic characteristics at different levels contributes to identifying various molecular subtypes, which can thereby shed new light on tumor clinical and mechanical differences, and reveal the potential biomarkers and therapeutic targets (Woo et al., 2017). Nonetheless, illustrating the complex STAD genomic data is still challenging. In this study, CNVcor and METcor gene dysregulation genomically and epigenetically was determined 
through the TCGA database. It is a strategy to integrate genomic and epigenomic data at different levels (Yeoh and Tan, 2021). According to our results, the aforementioned correlated genes contributed to identifying STAD subtypes, which reflected different molecular characteristics and genes correlated with immunity at different aspects, and prognostic outcomes. In addition, STADs that had increased frequency of abnormal CNVcor were associated with increased aberrant METcor frequency, which indicated the increased aberrant DNA MET frequency among cases showing DNA CNV frequency. The aforementioned results indicated that it was necessary to consider the aberrant DNA CNVs and MET during data analysis.

Fortunately, the current comprehensive analysis using CNVcor and METcor genes identified the new critical molecular features indicating novel STAD biomarkers and therapeutic targets. Analysis results of mutation types across different subtypes indicated that the differences in these 10 genes were significant in terms of the mutation frequency. Particularly, genes in iC1 subtype with optimal prognosis were associated with the gene mutation frequency. Five of the above 10 genes were reported to participate in STAD progression, pathogenesis, immune microenvironment, and malignant transformation, which were PIK3CA, SPECC1, ARID1A, ZBTB20, and KMT2D (Wang et al., 2011; Shi et al., 2017; Xiong et al., 2018; Ashizawa et al., 2019; Chen et al., 2019; Seo et al., 2019), and were related to the prognosis and survival for patients. Based on the aforementioned results, the bioinformatic mining results were highly reliable and accurate. Nonetheless, the associations of the five other genes with STAD have not been verified in fundamental or clinical study, which is the point that we are interested in. Among these five genes, ZFHX3 and RANBP2 are associated with an alternative mutation frequency among various malignant tumors (such as endometrial cancer and prostate cancer) (Packham et al., 2015; Walker et al., 2015; Hu et al., 2019). Nonetheless, it is still unknown about the underlying mechanism for modulating cancer genesis and development. Besides, the association of gene expression and mutation frequencies with STAD remains unknown. In this study, our results indicated that mutations in RANBP2 and PIK3CA partially mediated the favorable prognosis of STAD patients in iC1 subtype. Nonetheless, further protein-protein interaction analysis and molecular biological experiments are needed to verify these results.

Furthermore, JPH3, PLCXD3, and KCNB1 are the possible crucial regulating factors in the initiation and development of STAD. KCNB1 (Kv2.1), a major voltage-gated potassium channel $(\mathrm{Kv})$, is recognized to be the new prognostic factor to predict the survival for some cancers, including glioma, and it plays a tumorsuppressing role via inducing autophagy (Wang et al., 2016; Wang et al., 2017). JPH3 belongs to a member of junctional membrane complex (JMC) protein family, which can stabilize the JMC between plasma membrane and endoplasmic reticulum, and maintaining the cellular ultrastructure between intracellular ionic channels and cell surface. JPH3 is recognized as the new tumor suppressor gene with methylation within colorectal cancer, thus enhancing apoptosis mediated by mitochondria. In addition, it is the candidate biomarker for survival and metastasis for digestive tract cancers (Coleman et al., 2015; Hu et al., 2017). Yet, further studies are needed to illustrate the role of PLCXD3 in STAD. Our results suggested that expression of JPH3, PLCXD3, and KCNB1 showed marked correlation with DNA MET.

\section{CONCLUSION}

To sum up, the current comprehensive analysis based on gene expression at genomic and epigenomic levels reveals coordinated STAD genomic alternations at distinct points of view. Our results facilitate to determine the STAD molecular subtypes and to reveal accurate STAD clinical and mechanical diagnosis and treatments.

\section{DATA AVAILABILITY STATEMENT}

All data utilized in this work can be accessed from the corresponding author upon request. Source code is available at: https://github.com/code1234554321/code.git

\section{AUTHOR CONTRIBUTIONS}

JC and KY conceived and designed the experiments; JC, WL, and JD performed the experiments and wrote the paper; JC, PW, and JW analyzed the data. All authors have read and approved the final article.

\section{ACKNOWLEDGMENTS}

Our thanks should go to all members of the laboratory and collaborators for the assistance.

\section{SUPPLEMENTARY MATERIAL}

The Supplementary Material for this article can be found online at: https:/www.frontiersin.org/articles/10.3389/fgene.2021.778095/ full\#supplementary-material

Supplementary Figure S1 | 20 cluster iterations at $\mathrm{K}=2$ (category 3) were conducted for assessing iCluster cluster findings.

Supplementary Figure $\mathbf{S 2} \mid 20$ cluster iterations at $\mathrm{K}=3$ (category 4) were carried out for assessing the iCluster cluster findings.

Supplementary Figure S3 | Expression profiles of iCluster-identified subtypes. CNVcor (A) or METcor (B) gene-identified subtypes by NMF cluster approach are denoted in colored bars.

Supplementary Figure S4 | Comparison of overall survival of STAD patients in iC3 and iC1 (A) or iC2 (B) subtypes.

Supplementary Figure S5|T (A), N (B), M (C), stage (D), grade (E), and age (F) of STAD patients across the 3 subtypes were compared.

Supplementary Figure S6 | The KCNB1 (A) or JPH3 (B) levels in GSE62254 are classified as low and high groups ( $L 1-L 2)$, and the Kaplan-Meier curves for $L 1$ and L2 groups are displayed.

Supplementary Figure $\mathbf{S 7}$ | The gene expression levels of JPH3 in other GEO cohorts are classified as low and high groups, and Kaplan-Meier curves for these two groups are displayed. 
Supplementary Figure s8 I The relationship between KCNB1 (A), PLCXD3 (B), and JPH3 (C) levels and infiltrating degrees of immune cells within TME.

Supplementary Table S1 | Basic clinical information of STAD samples in TCGA.

Supplementary Table S2 | Basic information of CNVcor genes.

Supplementary Table S3 | Basic information of METcor genes.

Supplementary Table S4 | CNVcor gene distribution in diverse chromosomes.

Supplementary Table S5 | METcor gene distribution in diverse chromosomes.

Supplementary Table S6 | Sample distribution in different subtypes discovered from iCluster analysis or METcor and CNVcor genes.

\section{REFERENCES}

Arakawa, N., Sugai, T., Habano, W., Eizuka, M., Sugimoto, R., Akasaka, R., et al. (2017). Genome-wide Analysis of DNA Copy Number Alterations in Early and Advanced Gastric Cancers. Mol. Carcinog. 56 (2), 527-537. doi:10.1002/mc. 22514

Ashizawa, M., Saito, M., Min, A. K. T., Ujiie, D., Saito, K., Sato, T., et al. (2019). Prognostic Role of ARID1A Negative Expression in Gastric Cancer. Sci. Rep. 9 (1), 6769. doi:10.1038/s41598-019-43293-5

Balgkouranidou, I., Matthaios, D., Karayiannakis, A., Bolanaki, H., Michailidis, P., Xenidis, N., et al. (2015). Prognostic Role of APC and RASSF1A Promoter Methylation Status in Cell Free Circulating DNA of Operable Gastric Cancer Patients. Mutat. Research/Fundamental Mol. Mech. Mutagenesis 778, 46-51. doi:10.1016/j.mrfmmm.2015.05.002

Bray, F., Ferlay, J., Soerjomataram, I., Siegel, R. L., Torre, L. A., and Jemal, A. (2018). Global Cancer Statistics 2018: GLOBOCAN Estimates of Incidence and Mortality Worldwide for 36 Cancers in 185 Countries. CA: a Cancer J. clinicians 68 (6), 394-424. doi:10.3322/caac.21492

$\mathrm{Bu}, \mathrm{Z}$, and Ji, J. (2013). Controversies in the Diagnosis and Management of Early Gastric Cancer. Chin. J. Cancer Res. 25 (3), 263-266. doi:10.3978/j.issn.10009604.2013.06.15

Cai, L., Li, L., Ren, D., Song, X., Mao, B., Han, B., et al. (2020). Prognostic Impact of Gene Copy Number Instability and Tumor Mutation burden in Patients with Resectable Gastric Cancer. Cancer Commun. 40 (1), 63-66. doi:10.1002/cac2.12007

Chen, L.-h., Wang, L.-p., and Ma, X.-q. (2019). Circ_SPECC1 Enhances the Inhibition of miR-526b on Downstream KDM4A/YAP1 Pathway to Regulate the Growth and Invasion of Gastric Cancer Cells. Biochem. biophysical Res. Commun. 517 (2), 253-259. doi:10.1016/j.bbrc.2019.07.065

Choi, S. J., Jung, S. W., Huh, S., Chung, Y.-S., Cho, H., and Kang, H. (2017). Alteration of DNA Methylation in Gastric Cancer with Chemotherapy. J. Microbiol. Biotechnol. 27 (8), 1367-1378. doi:10.4014/jmb.1704.04035

Coleman, E. A., Lee, J. Y., Erickson, S. W., Goodwin, J. A., Sanathkumar, N., Raj, V. R., et al. (2015). GWAS of 972 Autologous Stem Cell Recipients with Multiple Myeloma Identifies 11 Genetic Variants Associated with ChemotherapyInduced Oral Mucositis. Support Care Cancer 23 (3), 841-849. doi:10.1007/ s00520-014-2406-x

Cristescu, R., Lee, J., Nebozhyn, M., Kim, K.-M., Ting, J. C., Wong, S. S., et al. (2015). Molecular Analysis of Gastric Cancer Identifies Subtypes Associated with Distinct Clinical Outcomes. Nat. Med. 21 (5), 449-456. doi:10.1038/nm. 3850

Ebrahimi, V., Soleimanian, A., Ebrahimi, T., Azargun, R., Yazdani, P., Eyvazi, S., et al. (2020). Epigenetic Modifications in Gastric Cancer: Focus on DNA Methylation. Gene 742, 144577. doi:10.1016/j.gene.2020.144577

El-Arabey, A. A., Abdalla, M., and Abd-Allah, A. R. (2020). SnapShot: TP53 Status and Macrophages Infiltration in TCGA-Analyzed Tumors. Int. Immunopharmacology 86, 106758. doi:10.1016/j.intimp.2020.106758

Feng, Y., Shi, C., Wang, D., Wang, X., and Chen, Z. (2019). Integrated Analysis of DNA Copy Number Changes and Gene Expression Identifies Key Genes in Gastric Cancer. J. Comput. Biol. : a J. Comput. Mol. Cel. Biol. 27 (6), 877-887. doi:10.1089/cmb.2019.0149

Han, Q., Zhou, H., Xie, W., Sun, T., Wei, R., Nie, C., et al. (2020). Association between the Methylation of the STAT1 and SOCS3 in Peripheral Blood and
Supplementary Table S7 | The levels of DNA copy-number gain/loss together with DNA hypermethylation/hypomethylation of samples.

Supplementary Table S8 | Immune scores for STAD samples.

Supplementary Table S9 | CNVcor genes with marked difference between iC2 and $\mathrm{iC} 1$ subtypes were obtained through Fisher's exact test.

Supplementary Table S10 | METcor genes with marked difference between iC2 and iC1 subtypes were obtained through Fisher's exact test.

Supplementary Table S11 | DEGs of iC1/iC2 subtypes were acquired through DESeq2.

Supplementary Table S12 | Distinctly different mutant genes between iC1 and iC2 subtype were selected upon Fisher's test.

Gastric Cancer. J. Gastroenterol. Hepatol. 35 (8), 1347-1354. doi:10.1111/jgh. 15021

Hu, Q., Zhang, B., Chen, R., Fu, C., A, J., Fu, X., et al. (2019). ZFHX3 Is Indispensable for ER $\beta$ to Inhibit Cell Proliferation via MYC Downregulation in Prostate Cancer Cells. Oncogenesis 8 (4), 28. doi:10.1038/ s41389-019-0138-y

Hu, X., Kuang, Y., Li, L., Tang, H., Shi, Q., Shu, X., et al. (2017). Epigenomic and Functional Characterization of Junctophilin 3 (JPH3) as a Novel Tumor Suppressor Being Frequently Inactivated by Promoter CpG Methylation in Digestive Cancers. Theranostics 7 (7), 2150-2163. doi:10.7150/thno.18185

Karimi, P., Islami, F., Anandasabapathy, S., Freedman, N. D., and Kamangar, F. (2014). Gastric Cancer: Descriptive Epidemiology, Risk Factors, Screening, and Prevention. Cancer Epidemiology, Biomarkers \& Prevention : a Publication of the American Association for Cancer Research. Cancer Epidemiol. Biomarkers Prev. 23 (5), 700-713. doi:10.1158/1055-9965.epi-13-1057

Li, B., Severson, E., Pignon, J.-C., Zhao, H., Li, T., Novak, J., et al. (2016). Comprehensive Analyses of Tumor Immunity: Implications for Cancer Immunotherapy. Genome Biol. 17 (1), 174. doi:10.1186/s13059-016-1028-7

Love, M. I., Huber, W., and Anders, S. (2014). Moderated Estimation of Fold Change and Dispersion for RNA-Seq Data with DESeq2. Genome Biol. 15 (12), 550. doi:10.1186/s13059-014-0550-8

Oh, S. C., Sohn, B. H., Cheong, J.-H., Kim, S.-B., Lee, J. E., Park, K. C., et al. (2018). Clinical and Genomic Landscape of Gastric Cancer with a Mesenchymal Phenotype. Nat. Commun. 9 (1), 1777. doi:10.1038/s41467-018-04179-8

Packham, S., Warsito, D., Lin, Y., Sadi, S., Karlsson, R., Sehat, B., et al. (2015). Nuclear Translocation of IGF-1R via p150Glued and an Importin- $\beta$ /RanBP2dependent Pathway in Cancer Cells. Oncogene 34 (17), 2227-2238. doi:10.1038/ onc.2014.165

Peng, Y., Wu, Q., Wang, L., Wang, H., and Yin, F. (2020). A DNA Methylation Signature to Improve Survival Prediction of Gastric Cancer. Clin. Epigenet 12 (1), 15. doi:10.1186/s13148-020-0807-x

Seo, A. N., Kang, B. W., Bae, H. I., Kwon, O. K., Park, K. B., Lee, S. S., et al. (2019). Exon 9 Mutation of PIK3CA Associated with Poor Survival in Patients with Epstein-Barr Virus-Associated Gastric Cancer. Anticancer Res. 39 (4), 2145-2154. doi:10.21873/anticanres.13328

Shen, R., Mo, Q., Schultz, N., Seshan, V. E., Olshen, A. B., Huse, J., et al. (2012). Integrative Subtype Discovery in Glioblastoma Using iCluster. PloS one 7 (4) e35236. doi:10.1371/journal.pone.0035236

Shi, J., Li, W., and Ding, X. (2017). Assessment of the Association between ZBTB20 Rs9841504 Polymorphism and Gastric and Esophageal Cancer Susceptibility: a Meta-Analysis. Int. J. Biol. Markers 32 (1), e96-e101. doi:10.5301/jbm.5000231

Shi, J., Zhou, W., Zhu, B., Hyland, P. L., Bennett, H., Xiao, Y., et al. (2016). Rare Germline Copy Number Variations and Disease Susceptibility in Familial Melanoma. J. Invest. Dermatol. 136 (12), 2436-2443. doi:10.1016/j.jid.2016. 07.023

Shiratsu, K., Higuchi, K., and Nakayama, J. (2014). Loss of Gastric Gland Mucinspecific O-Glycan Is Associated with Progression of Differentiated-type Adenocarcinoma of the Stomach. Cancer Sci. 105 (1), 126-133. doi:10.1111/ cas. 12305

Song, P., Wu, L., and Guan, W. (2020). Genome-Wide Identification and Characterization of DNA Methylation and Long Non-coding RNA Expression in Gastric Cancer. Front. Genet. 11, 91. doi:10.3389/fgene.2020. 00091 
Sung, H., Ferlay, J., Siegel, R. L., Laversanne, M., Soerjomataram, I., Jemal, A., et al. (2021). Global Cancer Statistics 2020: GLOBOCAN Estimates of Incidence and Mortality Worldwide for 36 Cancers in 185 Countries. CA A. Cancer J. Clin. 71 (3), 209-249. doi:10.3322/caac.21660

Van Cutsem, E., Dicato, M., Geva, R., Arber, N., Bang, Y., Benson, A., et al. (2011). The Diagnosis and Management of Gastric Cancer: Expert Discussion and Recommendations from the 12th ESMO/World Congress on Gastrointestinal Cancer, Barcelona, 2010. Ann. Oncol. 22 (Suppl. 5), v1-9. doi:10.1093/annonc/ mdr284

Walker, C. J., Miranda, M. A., O’Hern, M. J., McElroy, J. P., Coombes, K. R., Bundschuh, R., et al. (2015). Patterns of CTCF and ZFHX3 Mutation and Associated Outcomes in Endometrial Cancer. J. Natl. Cancer Inst. 107. doi:10. 1093/jnci/djv249

Wang, H.-Y., Li, J.-Y., Liu, X., Yan, X.-Y., Wang, W., Wu, F., et al. (2016). A Three Ion Channel Genes-Based Signature Predicts Prognosis of Primary Glioblastoma Patients and Reveals a Chemotherapy Sensitive Subtype. Oncotarget 7 (46), 74895-74903. doi:10.18632/oncotarget.12462

Wang, H.-Y., Wang, W., Liu, Y.-W., Li, M.-Y., Liang, T.-Y., Li, J.-Y., et al. (2017). Role of KCNB1 in the Prognosis of Gliomas and Autophagy Modulation. Sci. Rep. 7 (1), 14. doi:10.1038/s41598-017-00045-7

Wang, K., Kan, J., Yuen, S. T., Shi, S. T., Chu, K. M., Law, S., et al. (2011). Exome Sequencing Identifies Frequent Mutation of ARID1A in Molecular Subtypes of Gastric Cancer. Nat. Genet. 43 (12), 1219-1223. doi:10.1038/ng.982

Woo, H. G., Choi, J.-H., Yoon, S., Jee, B. A., Cho, E. J., Lee, J.-H., et al. (2017). Integrative Analysis of Genomic and Epigenomic Regulation of the Transcriptome in Liver Cancer. Nat. Commun. 8 (1), 839. doi:10.1038/ s41467-017-00991-w

Xiong, W., Deng, Z., Tang, Y., Deng, Z., and Li, M. (2018). Downregulation of KMT2D Suppresses Proliferation and Induces Apoptosis of Gastric Cancer. Biochem. biophysical Res. Commun. 504 (1), 129-136. doi:10.1016/j.bbrc.2018. 08.143

Xu, X., Chang, X., Xu, Y., Deng, P., Wang, J., Zhang, C., et al. (2020). SAMD14 Promoter Methylation Is Strongly Associated with Gene Expression and Poor
Prognosis in Gastric Cancer. Int. J. Clin. Oncol. 25 (6), 1105-1114. doi:10.1007/ s10147-020-01647-4

Ye, W., Held, M., Lagergren, J., Engstrand, L., Blot, W. J., McLaughlin, J. K., et al. (2004). Helicobacter pylori Infection and Gastric Atrophy: Risk of Adenocarcinoma and Squamous-Cell Carcinoma of the Esophagus and Adenocarcinoma of the Gastric Cardia. JNCI J. Natl. Cancer Inst. 96 (5), 388-396. doi:10.1093/jnci/djh057

Yeoh, K. G., and Tan, P. (2021). Mapping the Genomic Diaspora of Gastric Cancer. Nat. Rev. Cancer. [Epub ahead of print]. doi:10.1038/s41568-021-00412-7

Zeng, Z., Vo, A. H., Mao, C., Clare, S. E., Khan, S. A., and Luo, Y. (2019). Cancer Classification and Pathway Discovery Using Non-negative Matrix Factorization. J. Biomed. Inform. 96, 103247. doi:10.1016/j.jbi.2019.103247

Zhang, K., Gao, J., and Ni, Y. (2017). Screening of Candidate Key Genes Associated with Human Osteosarcoma Using Bioinformatics Analysis. Oncol. Lett. 14 (3), 2887-2893. doi:10.3892/ol.2017.6519

Conflict of Interest: The authors declare that the research was conducted in the absence of any commercial or financial relationships that could be construed as a potential conflict of interest.

Publisher's Note: All claims expressed in this article are solely those of the authors and do not necessarily represent those of their affiliated organizations, or those of the publisher, the editors, and the reviewers. Any product that may be evaluated in this article, or claim that may be made by its manufacturer, is not guaranteed or endorsed by the publisher.

Copyright (C) 2022 Chen, Liu, Du, Wang, Wang and Ye. This is an open-access article distributed under the terms of the Creative Commons Attribution License (CC BY). The use, distribution or reproduction in other forums is permitted, provided the original author(s) and the copyright owner(s) are credited and that the original publication in this journal is cited, in accordance with accepted academic practice. No use, distribution or reproduction is permitted which does not comply with these terms. 